\title{
A systematic review of non-pharmacological interventions used for pain relief after orthopedic surgical procedures
}

\author{
MEIFEN FAN and ZHEYING CHEN \\ Department of Operating Room, Renji Hospital, School of Medicine, \\ Shanghai Jiao Tong University, Shanghai 200127, P.R. China
}

Received October 3, 2019; Accepted July 21, 2020

DOI: 10.3892/etm.2020.9163

\begin{abstract}
The purpose of the present review was to evaluate the available evidence on the efficacy of various non-pharmacological interventions to relieve pain after orthopedic surgical procedures. An electronic search of the PubMed, Embase and Cochrane library databases was performed to retrieve studies of all types assessing the role of non-pharmacological interventions for pain relief after orthopedic surgical procedures. The included studies were required to assess pain outcomes using a validated measurement index, such as the Visual Analog Scale. The quality of randomized control trials (RCTs) was assessed using the Cochrane tool, while the ROBINS-I tool was used for non-RCTs. A total of five studies were included, namely three RCTs and two non-RCTs. The included studies used relaxation therapy, guided imagery, music and audio-visual distraction for pain management. There was considerable heterogeneity concerning study participants and types of intervention, which precluded a meta-analysis. Overall, all studies reported a significant beneficial effect of non-pharmacological interventions for pain relief. To conclude, current evidence from a limited number of studies indicates there may be a potential role of non-pharmacological interventions, including relaxation therapy, guided imagery, music and audio-visual distraction, in pain management of patients after orthopedic surgery. Owing to considerable heterogeneity and risk of bias in the included studies, strong conclusions cannot be drawn. Further high-quality RCTs assessing the role of such non-pharmacological techniques of pain management are required to strengthen the current evidence.
\end{abstract}

Correspondence to: Dr Zheying Chen, Department of Operating Room, Renji Hospital, School of Medicine, Shanghai Jiao Tong University, 160 Pujian Road, Shanghai 200127, P.R. China

E-mail: dr_zychen@163.com

Key words: pain, complementary therapy, surgery, music, relaxation therapy

\section{Introduction}

Orthopedic procedures are known to be painful to patients (1). In a comparison of pain intensity of surgical procedures, 22 of the 40 medical procedures with the highest pain level were orthopedic or trauma procedures of the extremities (2). Pain control is an important element of post-operative care as severe postoperative pain may increase the risk of complications, progress to persistent pain states and also delay rehabilitation (3). Post-operative pain may also increase overall healthcare costs due to a prolonged hospital stay and elevated rates of hospital readmission (4). Clinicians have reportedly used various means of pain control, including opioids, non-steroidal anti-inflammatory drugs (NSAIDs), local anesthetic infiltration and nerve blocks, for managing the analgesic requirements after orthopedic surgery (5). It is not surprising that orthopedic surgeons prescribe more opioids than any other surgical division (6).

A vital constituent of the pain management team is orthopedic nurses who spend considerable post-operative time with patients. Nursing personnel have an important role in patient preparation and management of the patients' pain and anxiety (7). They are not only responsible for the prescribed drug administration but may also support pain management by a variety of complementary therapies $(8,9)$. Several studies and reviews have assessed the role of complementary therapy, including listening to music, media distraction, relaxation therapy and guided imagery in reducing procedural or post-operative pain in a variety of patients (7-13). However, the literature on the use of such complementary therapies for orthopedic patients is limited. There is a requirement to provide evidence on this subject, e.g. by a pooled data synthesis, to guide nursing personnel on the exact role of these therapies in orthopedic patients. Therefore, the purpose of the present review was to assess the evidence on the efficacy of various non-pharmacological interventions to relieve pain after orthopedic procedures.

\section{Materials and methods}

Search strategy. An electronic search of the PubMed, Embase and Cochrane library databases was performed by two independent reviewers (MF and ZC). The last search was carried out on 1st July 2019. The following terms were employed 
for theliterature search: 'orthopedics', 'wounds', 'injuries', 'fracture', 'music', 'video', 'guided imagery', 'relaxation' and 'breathing exercises'. Further details regarding the search are outlined in Table SI. References of included studies were screened for the identification of any further relevant trials. The search results of all databases were initially evaluated by their titles and abstracts. Relevant articles identified by the initial screening were further scanned by their full-texts for inclusion in the present review. Disagreements between the two reviewers were resolved by discussion. This review was conducted following the guidelines of the Preferred Reporting Items for Systematic Reviews and Meta-analyses statement (14) and the Cochrane Handbook for Systematic Reviews of Intervention (15), except for protocol registration.

Inclusion criteria. All types of studies conducted on patients undergoing any orthopedic surgical procedure wherein patients were to receive a non-pharmacological intervention for postoperative pain management with or without a control group were included. The study aimed to assess pain outcomes using a validated measurement index, such as the Visual Analog Scale (VAS). Studies using non-pharmacological interventions with regional anaesthesia were excluded. Studies published in a language other than English, case reports, conference abstracts and review articles were also excluded. Conference abstracts were not included in the present study due to incomplete information available from such abstracts. The entire methodology was not clear and according to our experience, in the majority of cases, no response was received when contacting the corresponding authors for clarifications or for missing data.

Data extraction and outcomes. The two authors (MF and ZC) independently performed the extraction of available data using the pre-tested standardized format. Details extracted were as follows: First author, publication year, study design, country, demographic details, orthopedic surgery performed, intervention arms, sample size, assessment schedule, tools and interpretations, and results.

Quality assessment of studies and meta-analysis. The quality of randomized controlled trials (RCTs) was assessed using the Cochrane Collaboration risk assessment tool (15). The risk of bias in non-randomised studies of interventions (ROBINS-I) tool was used for quality assessment of non-randomized studies (16). Due to considerable heterogeneity in the interventions amongst the included studies, along with the difference in study types, a meta-analysis was not conducted and results are presented in a descriptive fashion.

\section{Results}

Selection of studies. A flow chart depicting the study retrieval and selection process is presented in Fig. 1. Of the relevant studies identified, five studies were finally included in the present review (17-21) and six studies were excluded (22-27). Details of included studies are presented in Table I and a list of excluded studies with reasons is presented in Table II. A total of three studies were RCTs $(17,18,21)$, while two were non-randomized single-arm studies $(19,20)$. All studies were performed at a single centre. The patient population in the included studies was not coherent. In addition, different non-pharmacological interventions were used in the included studies, including relaxation therapy, guided imagery, music and audio-visual distraction. A detailed description of each study is presented below.

Descriptive analysis of RCTs. Büyükyilmaz and Aşti (17), in an RCT on patients undergoing total hip or knee arthroplasty, assessed the role of relaxation techniques and back massage in reducing pain scores. They randomized their sample into intervention and control arms with 30 patients in each group. The relaxation techniques included rhythmic respiration, muscle relaxation exercises and listening to music. Patients also received a 10-min back massage lying on the intact hip/knee joint in a lateral position on their bed. The pain was measured before, immediately after, and one hour and two hours after therapy. The study reported a statistically significant reduction of pain in the intervention arm as compared to the control arm at all follow-up time-points.

Charette et al (18), in an RCT on patients who had received spinal fusion, evaluated the role of guided imagery and relaxation exercises in reducing pain scores. A total of 40 adolescent patients were randomly divided into two groups of 20 each. Patients in the intervention arm were provided with a DVD with information and guided imagery/relaxation exercises to practice at least three times a week at home. Pain scores were recorded pre-operatively, as well as at 14 and 30 days post-operatively. The authors reported a statistically significant reduction of pain at all follow-up time-points in the intervention group.

In another RCT, Elmali and Balci Akpinar (21) evaluated the effect of video distraction in reducing post-surgical pain scores in a sample of patients after orthopedic surgery. They randomized their sample into three groups of 30 patients each. The first group watched a comedy video, the second group watched an non-comedy video, while the third group served as a control. Pain scores were recorded on the VAS immediately and $30 \mathrm{~min}$ after the intervention. The authors reported a significant reduction of pain scores with the use of both comedy and non-comedy videos, while no such effect was seen in the control group. Pain reduction in both intervention groups indicated that video distraction rather than the comedy element may have served a role in the reduction of pain. Also, lack of blinding may have introduced bias in the overall results.

Descriptive analysis of non-RCTs. Schneider (20) conducted a single-arm study on 65 patients who had undergone varying orthopedic procedures. All patients listened to instrumental piano music for $35 \mathrm{~min}$. The music track selection was performed by a researcher who had a strong musical background and formal musical education. Pain scores were recorded prior to and just after the intervention. The authors reported a statistically significant reduction of pain scores after the intervention compared to pre-intervention levels.

Lim et al (19), in a single-arm study, assessed the efficacy of relaxation therapy in reducing pain in a cohort of patients with total knee replacement surgery. A total of 18 patients were included in their study. The intervention consisted of 


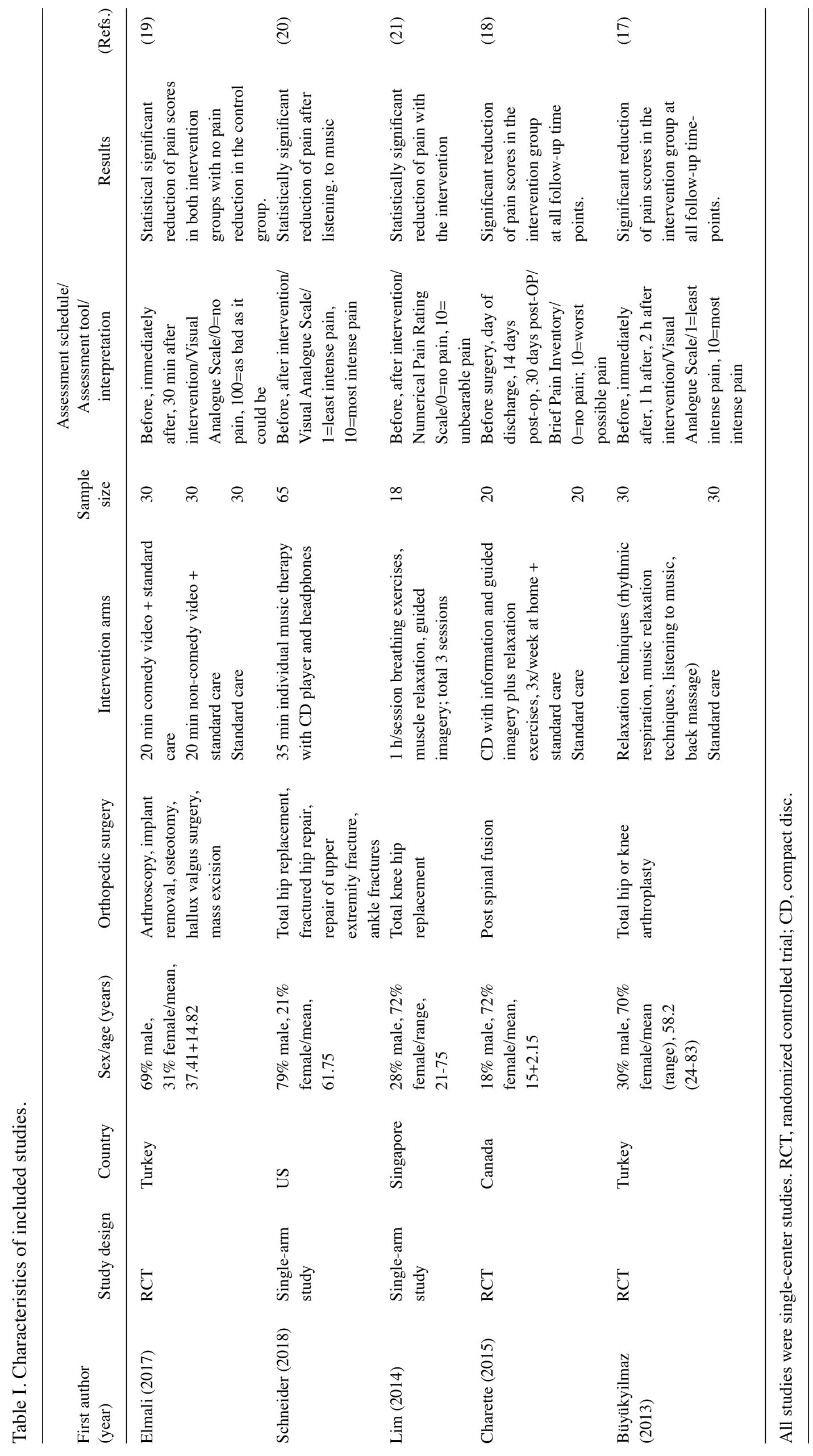


Table II. Excluded studies with reasons.

\begin{tabular}{ll}
\hline Study & \multicolumn{1}{c}{ Reason for exclusion } \\
\hline Chiodo et al (27) & Did not evaluate pain outcome \\
Tolunay et al (26) & Study not on orthopedic surgical patients \\
Athanassoglou et al (25) & Use of distraction with regional anesthesia \\
Hsu et al (24) & Did not evaluate pain outcome \\
Eckhouse et al (23) & Did not evaluate pain outcome \\
Rupérez Ruiz et al (22) & Use of distraction with regional anesthesia \\
\hline
\end{tabular}

three 1-h long individual-based sessions. All patients were first counseled about the negative effects of emotional tension and physical pain on postoperative recovery and the benefits of practicing the relaxation techniques. Following patient education, all patients practiced relaxation therapy consisting of breathing exercises with a background of soothing music and guided imagery. Pain scores were recorded prior to and after the intervention. The authors reported significantly reduced pain with the use of the intervention.

Quality of studies. The results of a quality assessment of the included studies are presented in Table III. A total of two RCTs $(18,21)$ had a low risk of bias due to randomization and allocation concealment. Due to the nature of the intervention, blinding was not possible. None of the trials was pre-registered. The summary risk of bias of the RCTs is presented in Fig. 2. The overall quality of non-RCTs was not high with high/unclear risk of bias across multiple domains (Table III).

\section{Discussion}

The present systematic review assessed evidence on the use of non-pharmacological interventions for pain management in patients undergoing orthopedic surgical procedures. The results of the present review indicated that several different strategies of non-pharmacological interventions have been used in orthopedic patients and all such complementary therapies may have certain benefits in the reduction of post-operative pain. The results are to be interpreted with caution, as only a small number of studies with considerable heterogeneity were included.

Non-pharmacological methods of pain management have gained popularity in the past decade owing to their ease of use and the side-effects associated with pharmacological interventions. The most commonly used pain medications after any surgical procedure are NSAIDs and opioids (5). Adverse effects of NSAIDs include gastric ulcers, bleeding complications and kidney injury (5). Specifically in orthopedic practice, the use of NSAIDs may inhibit fracture healing (28). Opioids, on the other hand, may have adverse effects including physical dependence, tolerance, respiratory depression, vomiting and constipation (29). In this context, it is important for clinicians as well as nursing personnel to identify non-pharmacological methods of pain management.

For the present review, five studies assessing different non-pharmacological techniques of pain control after orthopedic surgery were identified and included. A total of three of

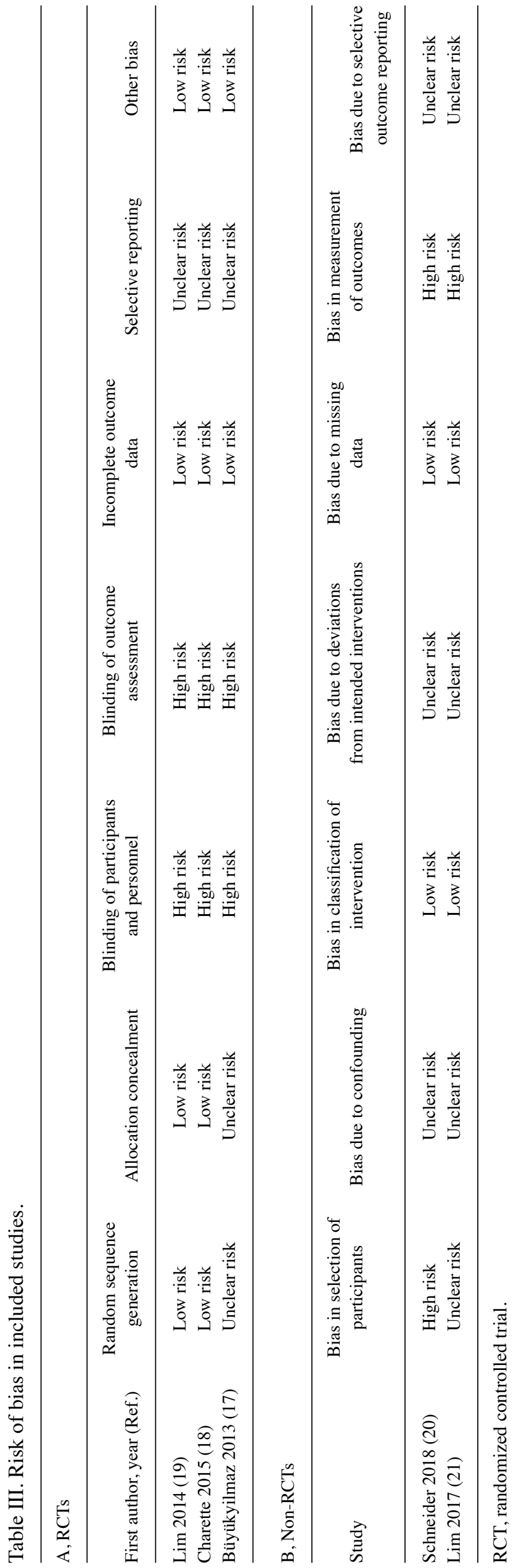



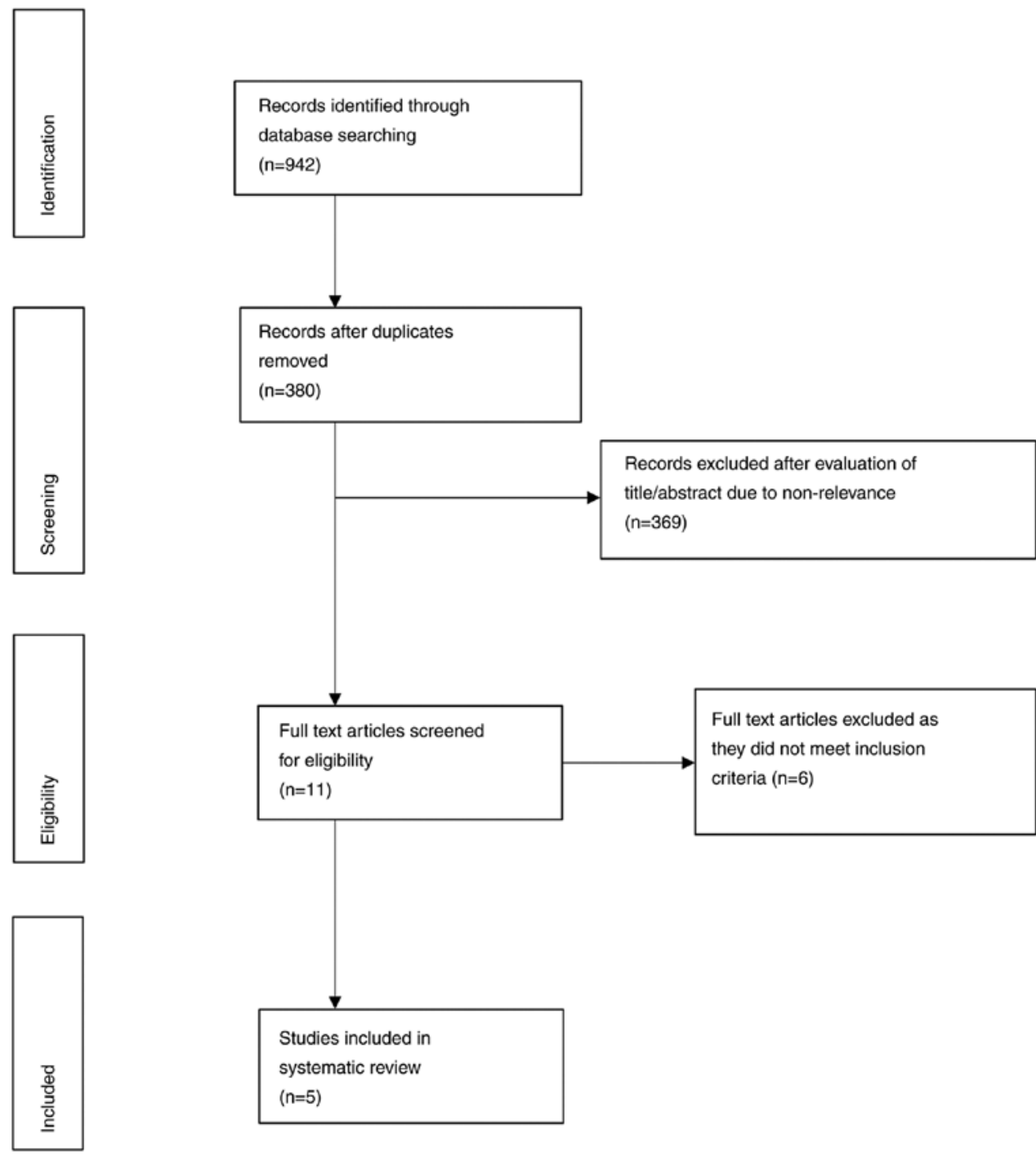

Figure 1. Flow chart for the search and selection of studies.

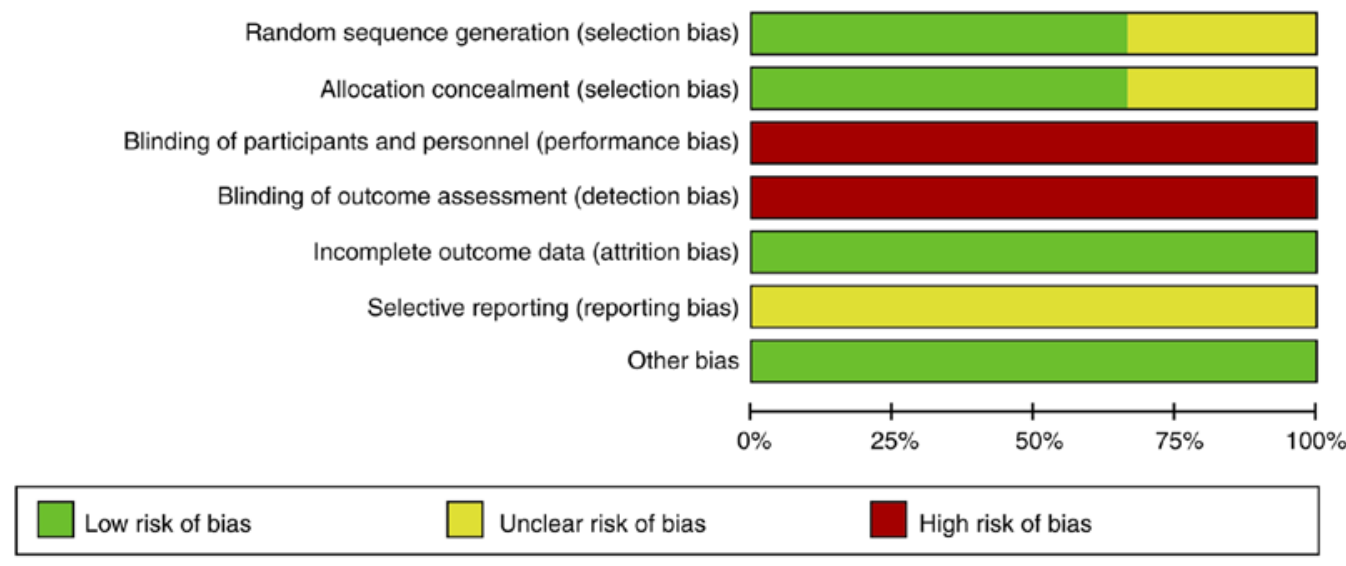

Figure 2. Overall risk of bias summary of randomized controlled trials.

the studies utilized relaxation therapy for pain control. It has been demonstrated that relaxation therapy is able to provide pain relief by decreasing anxiety, lowering muscle tension and distracting the patient (30). In a recent systematic review and meta-analysis of 12 studies, Ju et al (31) revealed that in patients undergoing abdominal surgery, relaxation therapy may achieve better pain relief as compared to standard treatment. In the present review, all three studies reported pain reduction with the use of relaxation therapy. It is important to note that out of the three studies, one was a single-arm study. 
In the absence of a control group, the actual beneficial effect of relaxation therapy in reducing pain cannot be validated. Also in the trial of Charette et al (18), guided imagery was used in combination with relaxation exercises. In guided imagery, mental images of pleasant sights, smells, sounds, tastes or other somatic sensations are used to generate a positive cognitive and emotional state in the patient (32). Due to the limited number of studies and the use of other non-pharmacological techniques with relaxation therapy, strong conclusions on the exact role of relaxation in the management of patients with orthopedic surgery cannot be drawn.

In two studies included in the present review, music and audiovisual distraction were used for pain management. The use of music or audio-visual media is one of the easiest distraction techniques and has been used in various medical disciplines. Song et al (10) performed a meta-analysis of nine RCTs, demonstrating significantly reduced pain with the use of music in patients undergoing minor surgical procedures. The use of audio-visual aids has been reported for pain management of pediatric dentistry patients (33), those with sickle cell disease (34) and patients undergoing colonoscopy (11). The trial of Elmali and Balci Akpinar (21) demonstrated that the use of comedy as well as non-comedy videos significantly reduced pain scores in patients following orthopedic surgery. On the other hand, the single-arm study of Schneider (20) also concluded that instrumental piano music may be helpful in reducing pain in the post-operative period.

The present review should be interpreted with the following limitations. First, only five studies were available for inclusion in the present review. Furthermore, there was significant heterogeneity amongst the included studies with respect to the patient population and the non-pharmacological intervention. This limited the feasibility of pooling the data for a meta-analysis. In addition, only three of the included studies were RCTs, while the other two were single-arm studies. Lack of a control group severely limits the ability to draw conclusions regarding the effectiveness of an intervention. As another limitation, the overall quality of studies assessed by the Cochrane tool and ROBINS-I tool was not high. There were numerous sources of bias in the included studies, for example lack of blinding, which weakens the conclusions that may be drawn from these trials. Finally, the studies included in the present review were restricted to those published in the English language, owing to constraints of translation. Furthermore, the present review did not include any studies published only as conference abstracts, owing to the limited data available from such publications.

To conclude, the present systematic review analyzed the role of non-pharmacological interventions in pain management provided to patients after orthopedic surgery. Current evidence from a limited number of studies indicates that there may be a potential role of non-pharmacological interventions, including relaxation therapy, guided imagery, music and audio-visual distraction, in the pain management of patients with orthopedic surgery. Owing to the considerable heterogeneity and risk of bias in the included studies, strong conclusions cannot be drawn. Further high-quality RCTs assessing the role of such non-pharmacological techniques of pain management are required to strengthen the current evidence.

\section{Acknowledgements}

Not applicable.

\section{Funding}

No funding received.

\section{Availability of data and materials}

The datasets used were from published studies.

\section{Authors' contributions}

MF conceived and designed the study. MF and ZC authors collected the data, performed the literature search and were involved in the writing of the manuscript. MF and $\mathrm{ZC}$ authors have read and approved the final manuscript.

\section{Ethics approval and consent to participate}

Not applicable.

\section{Patient consent for publication}

Not applicable.

\section{Competing interests}

The authors declare that they have no competing interests.

\section{References}

1. Barrington JW: Efficacy of periarticular injection with a long-acting local analgesic in joint arthroplasty. Am J Orthop (Belle Mead NJ) 44 (Suppl 10): S13-S16, 2015.

2. Gerbershagen HJ, Aduckathil S, van Wijck AJM, Peelen LM, Kalkman CJ and Meissner W: Pain intensity on the first day after surgery: A prospective cohort study comparing 179 surgical procedures. Anesthesiology 118: 934-944, 2013.

3. Gaffney CJ, Pelt CE, Gililland JM and Peters CL: Perioperative Pain Management in Hip and Knee Arthroplasty. Orthop Clin North Am 48: 407-419, 2017.

4. Hall G and Gregory J: The assessment and management of pain in an orthopaedic out-patient setting: A case study. Int J Orthop Trauma Nurs 22: 24-28, 2016.

5. Pasero $\mathrm{C}$ and McCaffery M: Orthopaedic postoperative pain management. J Perianesthesia Nurs 22: 160-174; quiz 172-173, 2007.

6. Koehler RM, Okoroafor UC and Cannada LK: A systematic review of opioid use after extremity trauma in orthopedic surgery. Injury 49: 1003-1007, 2018.

7. Brown MA: The role of nurses in pain and palliative care. J Pain Palliat Care Pharmacother 27: 300-302, 2013.

8. Zins S, Gross C and Hooke MC: Complementary therapies for pain among individuals receiving hemodialysis: A systematic review. Nephrol Nurs J 45: 13-23, 2018.

9. Aydin D, Sahiner NC and Ciftci EK: Non-pharmacological strategies used to reduce procedural pain in infants by nurses at family health centres. J Pak Med Assoc 67: 889-894, 2017.

10. Song M, Li N, Zhang X, Shang Y, Yan L, Chu J, Sun R and Xu Y: Music for reducing the anxiety and pain of patients undergoing a biopsy: A meta-analysis. J Adv Nurs 74: 1016-1029, 2018.

11. Xiaolian J, Xiaolin L and Lan ZH: Effects of visual and audiovisual distraction on pain and anxiety among patients undergoing colonoscopy. Gastroenterol Nurs 38: 55-61, 2015.

12. Drahota A, Galloway E, Stores R, Ward D, Severs M and Dean T: Audiovisual distraction as an adjunct to pain and anxiety relief during minor surgery. Foot (Edinb) 18: 211-219, 2008. 
13. Sahiner NC and Bal MD: The effects of three different distraction methods on pain and anxiety in children. J Child Health Care 20: 277-285, 2016.

14. Moher D, Liberati A, Tetzlaff J and Altman DG; PRISMA Group: Preferred reporting items for systematic reviews and meta-analyses: The PRISMA Statement. PLoS Med 6: e1000097, 2009.

15. Higgins J, Thomas J, Chandler J, Cumpston M, Li T, Page M and Welch V (eds.): Cochrane handbook for systematic reviews of interventions. Version 6. Cochrane, 2019. www.training. cochrane.org/handbook. Accessed, July 2019.

16. Sterne JA, Hernán MA, Reeves BC, Savović J, Berkman ND, Viswanathan M, Henry D, Altman DG, Ansari MT, Boutron I, et al: ROBINS-I: A tool for assessing risk of bias in non-randomised studies of interventions. BMJ 355: i4919, 2016.

17. Büyükyilmaz F and Aşti T: The effect of relaxation techniques and back Massage on pain and anxiety in Turkish total hip or knee arthroplasty patients. Pain Manag Nurs 14: 143-154, 2013.

18. Charette S, Fiola JL, Charest MC, Villeneuve E, Théroux J, Joncas J, Parent S and Le May S: Guided imagery for adolescent post-spinal fusion pain management: A pilot study. Pain Manag Nurs 16: 211-220, 2015.

19. Lim YC, Yobas P and Chen HC: Efficacy of relaxation intervention on pain, self-efficacy, and stress-related variables in patients following total knee replacement surgery. Pain Manag Nurs 15: 888-896, 2014

20. Schneider MA: The effect of listening to music on postoperative pain in adult orthopedic patients. J Holist Nurs 36: 23-32, 2018.

21. Elmali $\mathrm{H}$ and Balci Akpinar R: The effect of watching funny and unfunny videos on post-surgical pain levels. Complement Ther Clin Pract 26: 36-41, 2017.

22. Rupérez Ruiz MP, De San José I, Hermoso Montoya A, Ferreira Valencia T, Gómez Sanz A and López Gutiérrez A: Music therapy and regional anesthesia in orthopedic surgery. Rev Enferm 37: 27-30, 2014 (In Spanish).

23. Eckhouse DR, Hurd M, Cotter-Schaufele S, Sulo S, Sokolowski M and Barbour L: A randomized controlled trial to determine the effects of music and relaxation interventions on perceived anxiety in hospitalized patients receiving orthopaedic or cancer treatment. Orthop Nurs 33: 342-351, 2014.

24. Hsu CC, Chen WM, Chen SR, Tseng YT and Lin PC: Effectiveness of music listening in patients with total knee replacement during CPM rehabilitation. Biol Res Nurs 18: 68-75, 2016.
25. Athanassoglou V, Wallis A and Galitzine S: Audiovisual distraction as a useful adjunct to epidural anesthesia and sedation for prolonged lower limb microvascular orthoplastic surgery. J Clin Anesth 27 J Clin Anesth 27: 606-611, 2015.

26. Tolunay T, Bicici V, Tolunay H, Akkurt MO, Arslan AK, Aydogdu A and Bingol I: Rhythm and orthopedics: The effect of music therapy in cast room procedures, a prospective clinical trial. Injury 49: 593-598, 2018.

27. Chiodo CP, Keyser C, Palms D, Malone C, Bluman EM and Smith JT: The impact of music therapy in the postoperative setting on overall patient satisfaction using validated outcome scores. J Perianesthesia Nurs 34: 354-358, 2019.

28. Varghese D, Kodakat S and Patel H: Non-steroidal anti-inflammatories should not be used after orthopaedic surgery. BMJ 316 : 1390-1391, 1998

29. Viscusi ER and Pappagallo M: A review of opioids for in-hospital pain management. Hosp Pract (1995) 40: 149-159, 2012

30. Good M, Stanton-Hicks M, Grass JA, Cranston Anderson G, Choi C, Schoolmeesters LJ and Salman A: Relief of postoperative pain with jaw relaxation, music and their combination. Pain 81: 163-172, 1999.

31. Ju W, Ren L, Chen J and Du Y: Efficacy of relaxation therapy as an effective nursing intervention for post-operative pain relief in patients undergoing abdominal surgery: A systematic review and meta-analysis. Exp Ther Med 18: 2909-2916, 2019.

32. Quinn C: Guided imagery: An effective pain management tool for total joint replacement? Nursing (Lond) 49: 57-59, 2019.

33. Custódio NB, Costa FDS, Cademartori MG, da Costa VPP and Goettems ML: Effectiveness of virtual reality glasses as a distraction for children during dental care. Pediatr Dent 42: 93-102, 2020

34. Ezenwa MO, Yao Y, Engeland CG, Molokie RE, Wang ZJ, Suarez ML and Wilkie DJ: A randomized controlled pilot study feasibility of a tablet-based guided audio-visual relaxation intervention for reducing stress and pain in adults with sickle cell disease. J Adv Nurs 72: 1452-1463, 2016.

This work is licensed under a Creative Commons Attribution 4.0 International (CC BY 4.0) License. 